\title{
Development and applications of time-bunched and velocity-selected positron beams
}

Merrison, J.P.; Charlton, M.; Aggerholm, P.; Knudsen, H.; Werf, D.P. van der; Clarke, J.; Poulsen, Mogens Rysholt

Published in:

Review of Scientific Instruments

Link to article, DOI:

$10.1063 / 1.1581390$

Publication date:

2003

Document Version

Publisher's PDF, also known as Version of record

Link back to DTU Orbit

Citation (APA):

Merrison, J. P., Charlton, M., Aggerholm, P., Knudsen, H., Werf, D. P. V. D., Clarke, J., \& Poulsen, M. R. (2003). Development and applications of time-bunched and velocity-selected positron beams. Review of Scientific Instruments, 74(7), 3284-3292. https://doi.org/10.1063/1.1581390

\section{General rights}

Copyright and moral rights for the publications made accessible in the public portal are retained by the authors and/or other copyright owners and it is a condition of accessing publications that users recognise and abide by the legal requirements associated with these rights.

- Users may download and print one copy of any publication from the public portal for the purpose of private study or research.

- You may not further distribute the material or use it for any profit-making activity or commercial gain

- You may freely distribute the URL identifying the publication in the public portal 


\title{
Development and applications of time-bunched and velocity-selected positron beams
}

\author{
J. P. Merrison \\ Institute for Physics and Astronomy, Aarhus University, Aarhus-C, DK-8000, Denmark \\ M. Charlton \\ Department of Physics, University of Wales Swansea, Singleton Park, Swansea SA2 8PP, United Kingdom \\ P. Aggerholm and H. Knudsen \\ Institute for Physics and Astronomy, Aarhus University, Aarhus-C, DK-8000, Denmark \\ D. P. van der Werf and J. Clarke \\ Department of Physics, University of Wales Swansea, Singleton Park, Swansea SA2 8PP, United Kingdom \\ M. R. Poulsen \\ COM, Technical University of Denmark, Kgs. Lyngby DK-2800, Denmark
}

(Received 15 November 2002; accepted 4 April 2003)

\begin{abstract}
We describe the development of an instrument for the production of low energy positron beams that are bunched in time, and the use of a velocity selection device. The bunching unit was constructed from forty seven separate elements, coupled in series in a capacitor chain to reduce the delay time for propagation of the applied voltage pulse along the electrode system and to facilitate operation at frequencies up to $100 \mathrm{kHz}$. A parabolic potential distribution for time focusing was used. Tests with a dc positron beam produced from a radioactive source are described, together with measurements in which the buncher was used to compress positron pulses produced from an electron accelerator-based beam. Computer simulations of particle trajectories in the buncher have been performed resulting in a detailed evaluation of the factors that govern and limit the time resolution of the instrument. A sector magnet used to velocity-select intermediate energy positrons is described and its performance discussed. () 2003 American Institute of Physics. [DOI: 10.1063/1.1581390]
\end{abstract}

\section{INTRODUCTION}

The production of low energy positron beams (with energies typically from $\mathrm{eV}$ to $\mathrm{keV}$ ) has become a standard feature of many physics laboratories over the last 2 or 3 decades. ${ }^{1}$ There have been diverse applications for these beams, particularly in the areas of condensed matter and surface physics ${ }^{2,3}$ and atomic physics. ${ }^{4,5}$ Usually they are based around a radioactive $\beta^{+}$source such that the ensuing beam is continuous in nature (i.e., the positrons are randomly spaced in time). (Notable exceptions include beams based around pulsed machines, such as microtron electron accelerators and linear accelerators; see for example Ley ${ }^{6}$ for a summary and Mills et al. ${ }^{7}$ and Merrison et al. ${ }^{8}$ for discussions of other relevant instruments.) However, over the years there have been many successful attempts both to time $e^{9-12}$ and bunch $(\text { or pulse })^{13-16}$ radioactive source-based beams. The scientific motivation for doing so has been varied and includes the following; the measurement of positron lifetimes at surfaces ${ }^{11}$ and in the bulk ${ }^{16}$ of materials, atomic cross section measurements ${ }^{9}$ and the production of positronium beams, ${ }^{12}$ positronium spectroscopy, ${ }^{17,18}$ and the prospect of studying systems containing more than one positron. ${ }^{19-21}$

In this article we present details of a positron bunching system which has been applied, as described in Sec. II, both to continuous beams and to bunch the pulsed $(1 \mu$ s pulse time width) output of a microtron-based positron beam. ${ }^{8}$ The main novel feature of the system is that the electrical ele- ments of the buncher are capacitively coupled, thus reducing pulse propagation delay and allowing the device to be operated at frequencies much greater than has been achieved with similar resistively coupled devices. ${ }^{7}$

Several techniques have been used in conjunction with positron beams to provide energy analysis of the beam, or merely to deflect or guide the low energy positrons such that, for instance, higher energy particles emanating from the radioactive source do not contaminate the beam. A summary of these has been provided by Coleman ${ }^{1}$ and they include timeof-flight systems, ${ }^{9,10} \mathbf{E} \times \mathbf{B}$ velocity analyzers, ${ }^{22}$ bent solenoids, ${ }^{23}$ and electrostatic energy analyzers. ${ }^{24,25}$ Here we describe the construction and use of a simple magnetic sector field analyser for positron studies. The device is a true momentum analyzer (as opposed to kinetic energy), is simple to implement, and is especially suited for use with intermediate energy positron beams. Discussion of the instrument can be found in Sec. III.

\section{THE CAPACITANCE CHAIN BUNCHER}

\section{A. Mechanical and electrical construction}

The buncher consisted of 47 cylindrical elements each with an internal diameter of $50 \mathrm{~mm}$ and a length of $20 \mathrm{~mm}$. They were held separately by bolts onto three long $(>1 \mathrm{~m})$ ceramic rods. The separation between each element was fixed at $1 \mathrm{~mm}$ using ceramic spacers and the array was held 


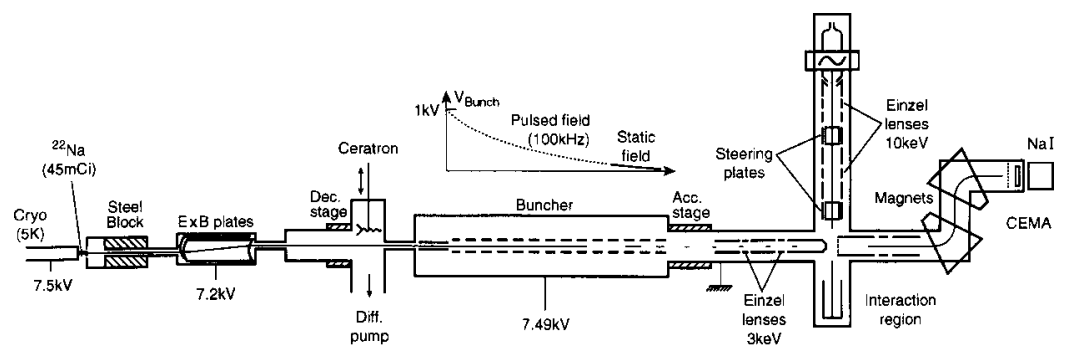

FIG. 1. Schematic of the experimental apparatus, not to scale. The $7.49 \mathrm{kV}$ shown applied to the buncher chamber was variable with respect to the voltage applied to the positron moderator, which was located on the $5 \mathrm{~K}$ cryohead. This difference set $V_{e}$, the positron kinetic energy in the buncher. The height of the buncher voltage, shown as $1 \mathrm{kV}$, could also be varied, as could the pulse repetition rate, with $100 \mathrm{kHz}$ being an upper limit. On the right of the diagram einzel lenses and a pair of magnetic sector field momentum analyzers are illustrated. For the buncher tests a ceratron electron multiplier detector replaced the einzel lens.

along the axis of the positron beamline (which was also the axis of the magnetic field used to confine the beam). The buncher elements were held inside a cylindrical vacuum chamber, which was incorporated into a positron beamline developed at the University of Aarhus. ${ }^{8,26}$ This setup is shown schematically in Fig. 1.

Each buncher element was wired to a separate vacuum feedthrough using copper wire. The feedthroughs were welded into a specially designed flange in two rows such that their position followed the physical layout of the buncher elements within the beamline chamber. Thus, the length of the wires to the feedthroughs was kept to a minimum, and each was approximately the same length. There was a distance of $38 \mathrm{~mm}$ between the two rows of feedthroughs and the diagonal distance from feedthrough-to-feedthrough was $58 \mathrm{~mm}$. The overall length of the capacitor chain was $2.21 \mathrm{~m}$, and using the speed of light in vacuum the minimum propagation delay down a chain of this length would be 7.4 ns. The measured value, between elements 1 and 33, for a pulse height (or buncher voltage $V_{b}$ ) of $300 \mathrm{~V}$ was $10 \mathrm{~ns}$. The rise time of the pulse, which was dominated by the performance of the pulse generator/amplifier used, was measured directly from an oscilloscope by estimating the total elapsed time for the pulse to rise from zero to its maximum height. The rise time was found to vary down the buncher chain falling approximately linearly from $30 \mathrm{~ns}$ for the first electrode to around $10 \mathrm{~ns}$ for electrode 33 . Hulett et al., ${ }^{27}$ who used a device similar to the buncher described here as an ionic mass spectrometer, have found from both a theoretical approach and direct measurement that the pulse rise time had very little influence upon the time focusing properties of their device. This is probably because their voltage distribution was still quadratic and therefore time focusing, 7,13,27 despite the fact that the amplitude of the voltage pulse changed with time. However, the situation here, with a variable rise time, results in a more complex behavior and further discussion of this can be found in Sec. II D.

The bunching was obtained by applying the pulsed voltage, $V_{b}$, to one end of the series capacitor chain, which was then divided in a parabolic fashion by the chain. In order to calculate the values of the capacitances to be inserted between adjacent feedthroughs (and hence electrodes), the intrinsic capacitance between each pair of electrodes had to be measured, along with the capacitance of each electrode to ground. (Although the electrodes were nominally the same size, small variations in mounting position and the distance of the gap between them meant that the pairwise capacitances were individually measured. Likewise, variations from electrode-to-electrode in their positions with respect to a grounded object, meant that the capacitance to ground of each electrode was individually accounted for.)

Figure 2(a) shows the effective capacitance network for the final two elements of the buncher system. The capacitances between the electrodes are denoted as $C_{n}$ and $C_{n-1}$, with the measured capacitance of the $n$th and $(n-1)$ th electrodes to ground denoted as $C_{n}^{g}$ and $C_{n-1}^{g}$, respectively. It is easy to find a recursion formula that relates the capacitances to the voltages that should be applied to each electrode (to form the parabolic array), and this is given by

$$
C_{n-1}=\left\{C_{n}\left(V_{n-1}-V_{n}\right)+C_{n-1}^{g} V_{n-1}\right\} /\left\{V_{n-2}-V_{n-1}\right\},
$$

where $C_{n}=C_{n}^{g} V_{n} /\left(V_{n-1}-V_{n}\right)$. Thus, once the respective $C_{n} \mathrm{~s}$ are known, and the intrinsic capacitance between each pair measured, the capacitance to be added can be calculated. A computer program was written to evaluate the relevant capacitances, and which also gave as output the voltage
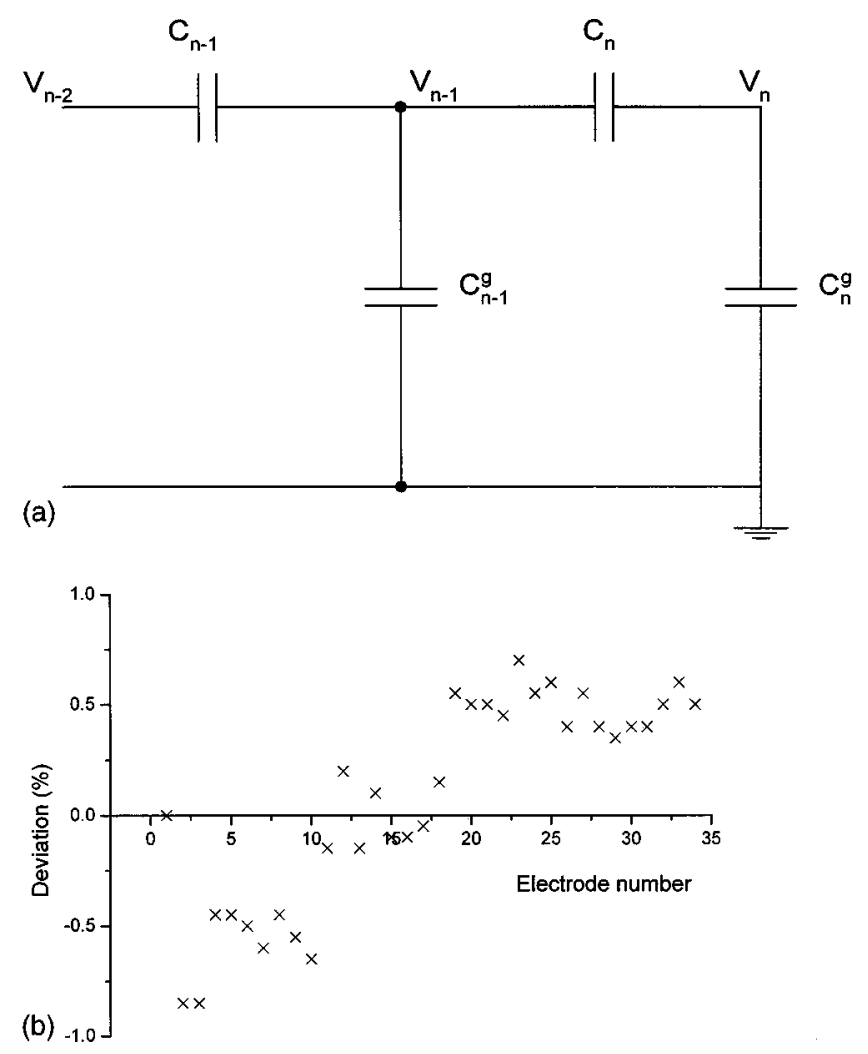

FIG. 2. (a) Electrical circuit showing capacitances of the final two elements of the buncher [see Eq. (1) and accompanying discussion]. (b) The measured deviation from the ideal quadratic potential for the real buncher. 
which would be measured at each electrode using a probe of known input capacitance. Figure 2(b) shows the voltage measured on each electrode expressed, for clarity in evaluating the accuracy of our method, in terms of the deviations from the expected parabolic distribution. The agreement obtained is excellent, and is everywhere between $\pm 1 \%$, although there is a systematic trend from negative to positive passing along the buncher from the high to the low voltages. The origin of this effect is not known.

\section{B. Tests using a continuous positron beam}

A continuous positron beam was generated using a 1.5 $\mathrm{GBq}^{22} \mathrm{Na}$ radioactive source and a solid argon moderator. ${ }^{1,2}$ The beam had a measured energy spread of around $5 \mathrm{eV}$ and a similar mean energy, although the latter could be altered by changing $V_{\mathrm{ff}}$, the potential with respect to ground applied to the vacuum chamber which housed the buncher. The positrons were confined by an axial magnetic field of around 5 $\mathrm{mT}$ and passed through the buncher, which was switched on at various controlled frequencies. In these tests the last 14 elements of the instrument were wired using a resistor chain and were dc biased. This provided a quadratic potential distribution across the last section of the buncher and, when the capacitor chain section of the instrument was off, returned positrons back along the length of the device. As such, the active length was twice the physical length of the capacitor chain section, or very nearly $1.4 \mathrm{~m}$. If a positron was present in the buncher when the pulse was applied it was ejected from the instrument and detected using a ceratron electron multiplier detector (not shown in Fig. 1). The efficiency, $\epsilon$, of bunching was measured by using standard timing (TAC/ MCA) electronics to register coincidences between the detected positron and the trigger pulse for the buncher. The efficiency was found by dividing the coincidence rate by the incident positron flux with the buncher off. Using these means also allowed the timing resolution of the device to be determined.

The efficiency of the buncher was expected to be the product of the time-of-flight of the positrons in the device, $t_{f}$, and the repetition rate of the pulse sequence, $f_{b}$, since the pulse occurs randomly with respect to the arrival times of positrons in the buncher itself. The measured efficiency versus buncher repetition rate is shown in Fig. 3(a). A straight line fit to the data yields a value for $t_{f}$ of $1.04 \pm 0.05 \mu \mathrm{s}$, and thus a mean energy of the positrons in the buncher of 5.1 $\pm 0.5 \mathrm{eV}$. This is in accord with the expectations, since for this experiment the buncher chamber was floated to the same electrical potential as the moderator bias such that the positron beam would only have its mean intrinsic kinetic energy once inside the device.

Figure 3(b) shows a plot of the bunching efficiency against $V_{\mathrm{fl}}$ (which effectively fixes the beam energy in the buncher) at a constant buncher frequency of $67.6 \mathrm{kHz}$. The measured efficiency rises steadily as the floating voltage is increased, except for the final point taken at around $38 \mathrm{~V}$, by which time part of the beam is being retarded off by the application of the voltage. The rising portion of the data were fitted to a function of the form $a /\left(b-V_{\mathrm{fl}}\right)^{1 / 2}$, with $a$ and $b$
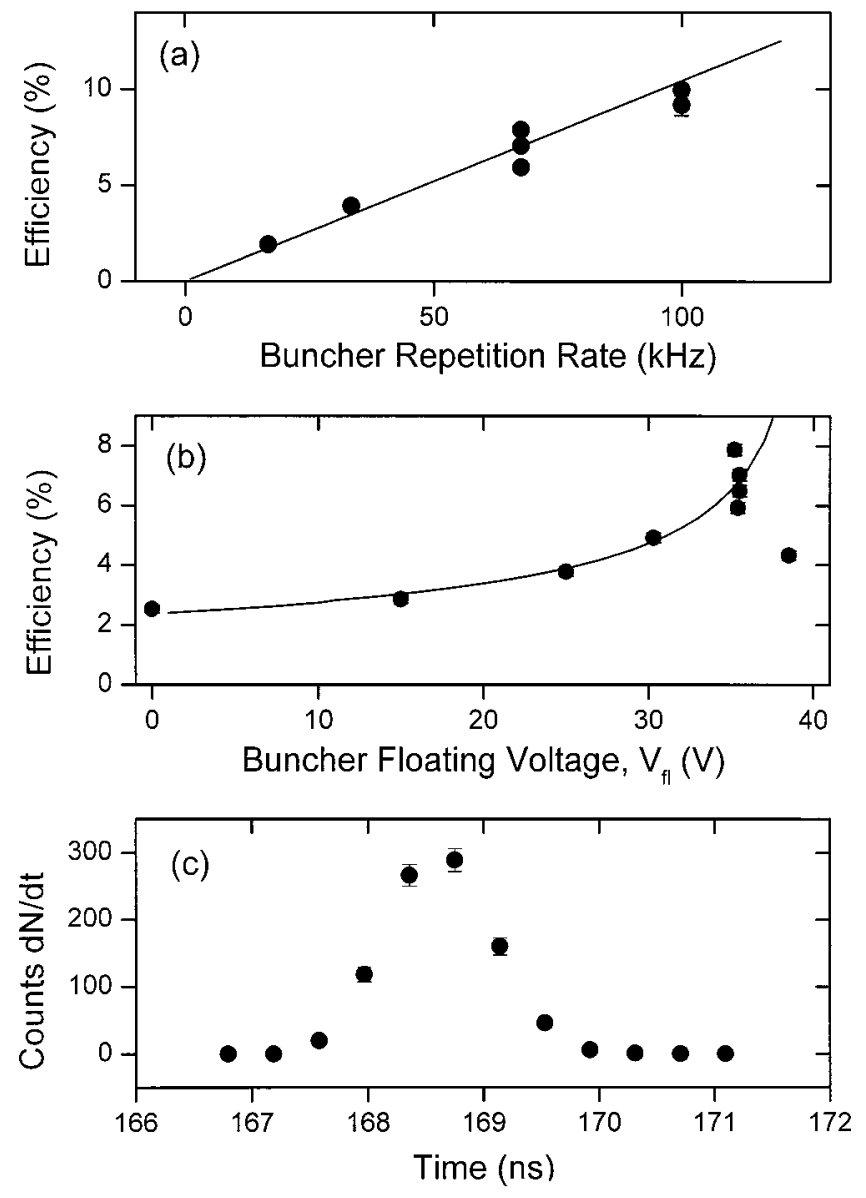

FIG. 3. (a) Buncher efficiency vs repetition rate for a continuous positron beam. (b) Buncher efficiency vs floating voltage with respect to ground $\left(V_{\mathrm{ff}}\right)$ at a fixed repetition rate of $67.6 \mathrm{kHz}$. See accompanying text for a discussion, including the significance of the fitted line. (c) Measured timing resolution for a bunched positron beam $\left(V_{b}=1 \mathrm{kV}\right)$.

constants, which yielded $a=15.3(1.6) \%$ and $\mathrm{b}=40.5$ (1.3) $\mathrm{eV}$. The analytic expectation, using the effective buncher length of $1.4 \mathrm{~m}$ to evaluate the time of flight of the positrons in the buncher [which is proportional to $1 /\left(V_{e}\right)^{1 / 2}$, where $V_{e}$ is the energy of the beam in the buncher] is $15.9(\%) /\left(V_{e}\right)^{1 / 2}$, in excellent accord with the measurements. The fitted constant $b$ is also in good accord with the expectation that it should be equal to the $35.2 \mathrm{~V}$ potential difference between the moderator and the buncher chamber when grounded, plus the mean intrinsic energy of the positrons of $5.1 \pm 0.5 \mathrm{eV}$. The variations in the measured efficiency near the floating voltage of $35 \mathrm{~V}$ probably reflect the changing nature of the surface of the solid argon moderator during the period over which the measurements were made [a similar effect can be seen in Fig. 3(a)]. At this voltage the beam is at a low kinetic energy inside the device and the efficiency will be very sensitive to surface changes which might alter the ejection energy of the positrons.

A measurement of the timing resolution taken at a value of the buncher pulse voltage $V_{b}=1 \mathrm{kV}$ is shown in Fig. 3(c). The value for the full width at half maximum (FWHM) of the distribution is approximately $1.2 \mathrm{~ns}$, while a result of 2.0 ns is obtained for the $10 \%-90 \%$ width. An estimate of the expected resolution can be found by solving the equations of 


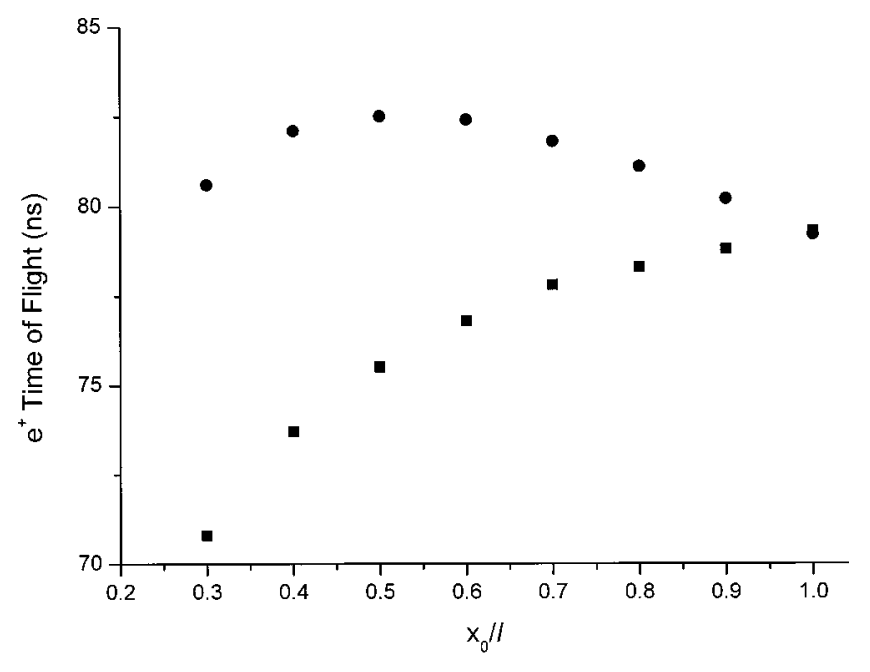

FIG. 4. Times-of-flight at various starting positions, $x_{0}$, in the buncher as deduced from Eqs. (2) (filled squares) and (3) (filled circles), with the latter incorporating a simple time shift in an attempt to allow for the finite pulse propagation delay down the length of the instrument. In this case $V_{b} / V_{e}$ $=200$.

motion for particles in a quadratic potential, allowing for their finite initial speed. The time taken for the positrons to reach the end of the buncher once it has been switched on is given by

$$
t\left(x_{0} / l\right)=\tan ^{-1}\left\{\left(V_{b} / V_{e}\right)^{1 / 2} x_{0} / l\right\} / \omega,
$$

where $\omega=\left(2 e V_{b} / m\right)^{1 / 2} / l, x_{0}$ is the starting position of the positron from the end of the buncher, and $l$ is the physical buncher length. Similar expressions can be found in the work of Hulett et $a .^{27}$ and Crane and Mills. ${ }^{13}$ Inserting values for our case, and with $\left(V_{b} / V_{e}\right)=200$, we find that the maximum flight time is $79.2 \mathrm{~ns}$ (corresponding to $x_{0} / l=1$ ) while the minimum value is $70.8 \mathrm{~ns}$ for $x_{0} / l=0.3$, the last portion of the buncher which is pulsed on. Thus, the expected time width $(8.4 \mathrm{~ns})$ is much broader than that measured. A potential cause of this difference is the finite propagation time of the pulse down the capacitor chain. In the ideal case, in which the positrons would be motionless in the buncher $\left(V_{\mathrm{e}}=0\right)$ when the voltage was applied, the time of flight would reduce from Eq. (2) to $\pi / 2 \omega$ and the pulse propagation time would only degrade the timing performance. In reality the positrons are not stationary and those located in the buncher at smaller values of $x_{0} / l$ are expected to reach the end of the buncher first. However, the pulse is applied first to the electrode corresponding to $x_{0} / l=1$, and is delayed in its application to elements further down the chain. This creates a time shift (delay) on moving down the buncher, which in our case amounted to around $10 \mathrm{~ns}$ by $x_{0} / l=0.3$ (electrode 33 ). A modified expression for the times-of-flight is then given by

$$
\begin{aligned}
t\left(x_{0} / l\right)= & \tan ^{-1}\left\{\left(V_{b} / V_{e}\right)^{1 / 2} x_{0} / l\right\} / \omega \\
& +14\left(1-x_{0} / l\right)(\mathrm{ns}) .
\end{aligned}
$$

Figure 4 shows a plot of the expected times-of-flight at various positions along the buncher and the effect of the time shift introduced by the pulse propagation time. The time width is shortened and is much closer to the measured value quoted above. Supporting evidence for a beneficial effect of the pulse propagation delay has been forthcoming from simulations of the buncher performance, as described in Sec. II D. However, there are further complexities associated with the temporal behavior of the electric field, and hence the acceleration of the positrons in the electrode structure, which must be taken into account. These include effects due to the finite propagation time of the pulse down the buncher and its rise time. Both of these effects were included in the trajectory simulations.

\section{Tests using a pulsed positron beam}

These tests were performed at the pulsed slow positron facility which has been developed at the Institute for Storage Ring Facilities at Aarhus (ISA). An account of this facility has been presented by Merrison et al., ${ }^{8}$ including a brief report of their preliminary bunching experiments. Here we provide a more complete account of the work.

The beamline produced $700 \mathrm{~ns}$ wide bursts containing around 30000 positrons at a frequency of $12 \mathrm{~Hz}$. These were transported to the buncher at kinetic energies of 200 or 500 $\mathrm{eV}$. At this facility it was necessary to use a tungsten foil to create the low energy positrons, ${ }^{8}$ resulting in an intrinsic energy spread of around $3 \mathrm{eV}$. Since this spread is much less than the transport energy, the time structure of the pulse was preserved at the input to the buncher. The output of the beamline and buncher was monitored at the exit of the buncher using a channel electron multiplier array (CEMA) detector. The output of this was $50 \Omega$ coupled to a 1.5 $\mathrm{GS} \mathrm{s}^{-1}, 400 \mathrm{MHz}$ bandwidth, oscilloscope, and the timing resolution was estimated by examining the pulse shape directly on the oscilloscope. Note that in these tests the full buncher was used such that the capacitor chain was extended to cover all 47 elements.

Figure 5 shows the output of the CEMA detector for four different operating conditions. For these experiments the beam was transported at $500 \mathrm{eV}$, while the buncher was floated at $495 \mathrm{~V}$. The buncher pulse height was $750 \mathrm{~V}$. Figure 5(a) shows the "background" registered by the CEMA with the positron beam off (which was due to electronic pickup from the facility microtron and the buncher) while Fig. 5(b) is the signal with the positron beam on, but the buncher off. Figure 5(c) is "beam-plus-background" with the buncher on and finally Fig. 5(d) is for the buncher on with the "background" removed. The buncher has transformed the second broad feature in Fig. 5(b) into the narrow spike seen in Figs. 5(c) and 5(d).

The time width of this peak was measured directly as outlined above for various operating conditions, and this was taken as a measure of the timing resolution of the instrument. However, this could only yield an upper limit estimate, since the measured time width would also be affected by the detector response, that of associated circuitry and that of the oscilloscope. Figure 6 shows data in which the timing response was measured at various pulsing voltages. The measured response falls with $V_{b}$, as expected from Eq. (2), and reaches a plateau, with a hint of a rise as the voltage approaches $800 \mathrm{~V}$. As noted above, the values given for the timing resolution are upper limits and are much larger than 


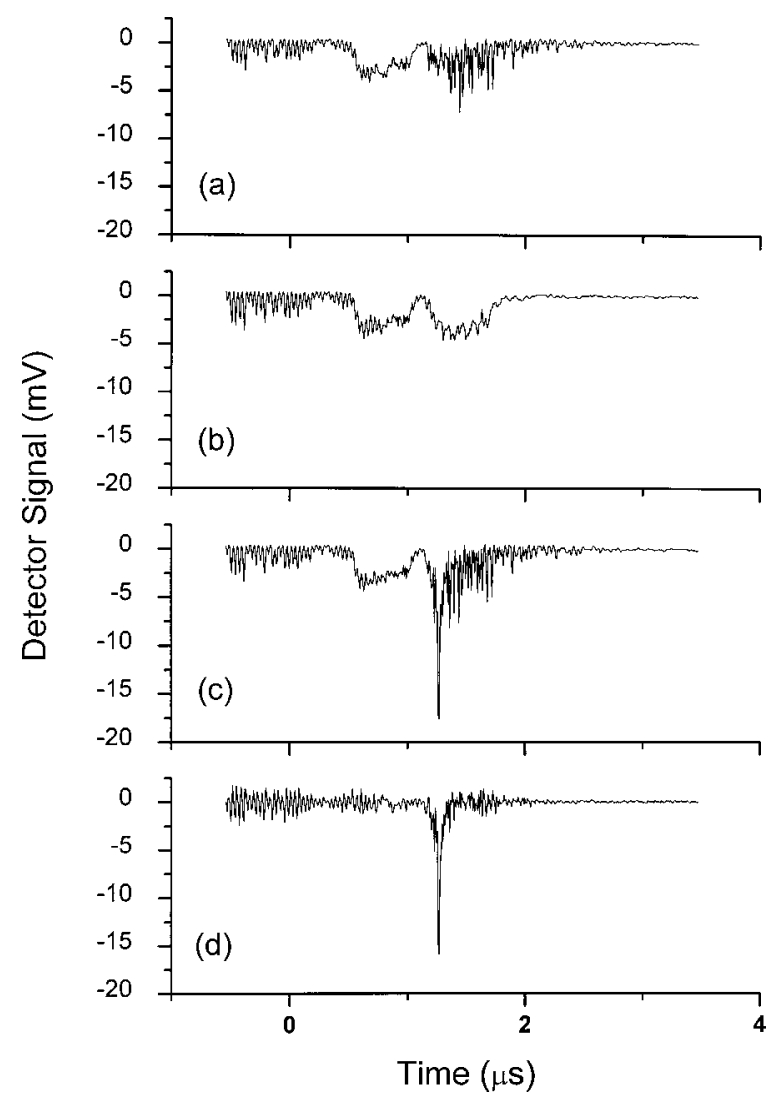

FIG. 5. Timing output of a CEMA detector as recorded on an oscilloscope for a bunched, pulsed low energy positron beam: (a) background with the beam off; (b) beam on, but the buncher off; (c) beam plus background with the buncher on; and (d) buncher and beam on, but background removed.

that found under similar conditions for the continuous beam. In the latter instance single particle counting techniques could be applied and a shorter active buncher length was used.

\section{Simulations}

The performance of the buncher was modelled using the SIMION V7.0 ion optics simulation program. ${ }^{28}$ This allowed

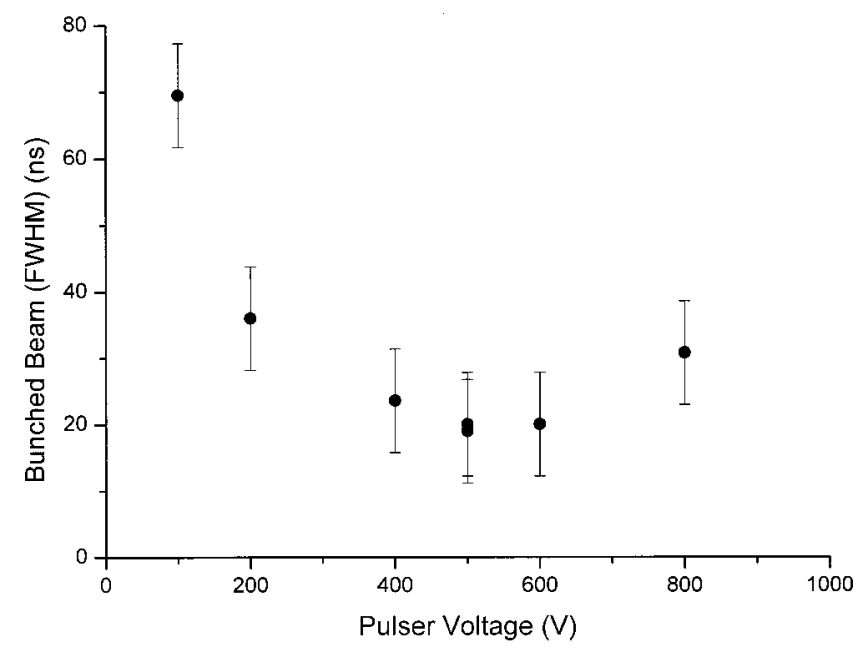

FIG. 6. Measured time response of the bunched, pulsed positron beam at various values of $V_{b}$, the pulser voltage. The uncertainties reflect the error with which the positron pulse time width could be estimated from the oscilloscope.

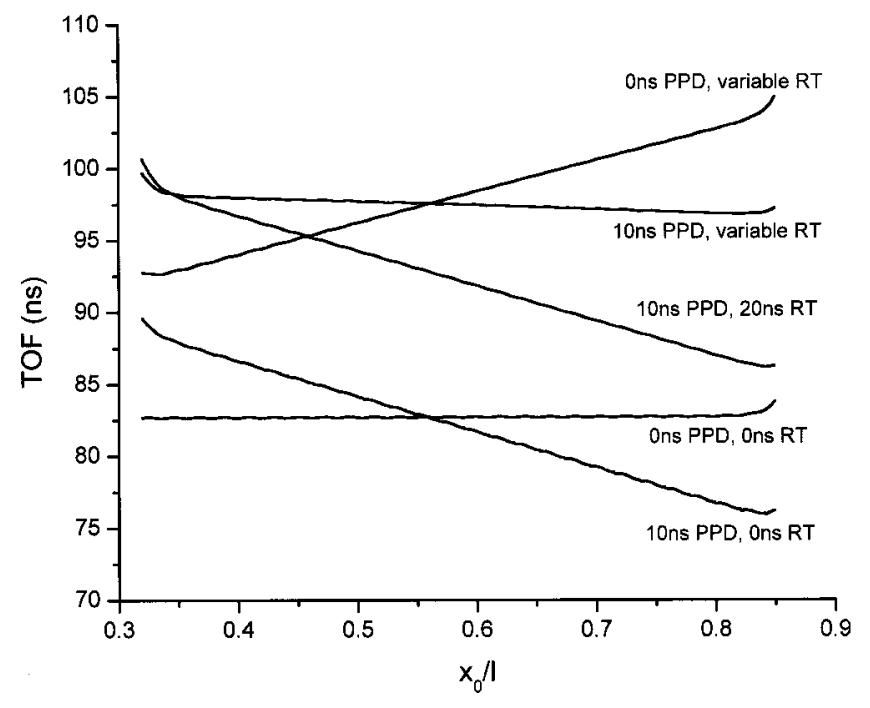

FIG. 7. Simulated times-of-flight at various starting points in the buncher for $V_{b}=1000 \mathrm{~V}$ and $V_{e}=0$ and at selected values of pulse propagation delay (PPD) and rise time (RT).

positron trajectories to be calculated for the real buncher (i.e., a system of discrete electrodes), rather than the idealized parabolic potential, for a range of positron energies within the instrument and for typical applied pulsed voltages. Thus, the behavior of a beam of finite energy spread in the true buncher potential distribution could be elucidated. It should be noted that the majority of our simulations were performed without inclusion of the axial magnetic field and with positrons considered to be moving along the axis of the instrument. However, selected work with magnetic field incorporated, and with off axis and angular motion included, showed that these did not have major effects on the simulated performance of the buncher. These will be discussed as appropriate. The positron times-of-flight were simulated to a point $50 \mathrm{~mm}$ beyond the center of the last electrode, since the detector was physically located at this point during the experiments.

Figure 7 shows the simulated times-of-flight versus starting position in the buncher for positrons at rest and $V_{b}$ $=1 \mathrm{kV}$ for five different combinations of pulse propagation delay and rise time. The simplest situation is the ideal case of zero pulse propagation and rise times. For a continuous parabolic potential this situation should, according to Eq. (2), lead to a perfect time focus. It is expected that this would be approached when $V_{b} \gg V_{e}$, which may, for instance, be the case for thermal positrons in an accumulator or trap-based beam, ${ }^{29,30}$ particularly if the length of the buncher occupied by the trapped particles is much smaller than $l$. However, Fig. 7 shows that there are small ripples on the times-offlight which are due to deviations from the parabolic potential form caused by the discrete electrodes. In our case the ripples are 50-100 ps in height, leading to a corresponding limit in timing resolution. This will be a general feature of all discrete electrode bunchers.

Figure 7 also shows the results of a simulation for the same conditions $\left(V_{b}=1 \mathrm{kV}, V_{e}=0 \mathrm{eV}\right)$, but now with the incorporation of the pulse rise time and the pulse propagation delay incremented appropriately down the buncher. The ef- 


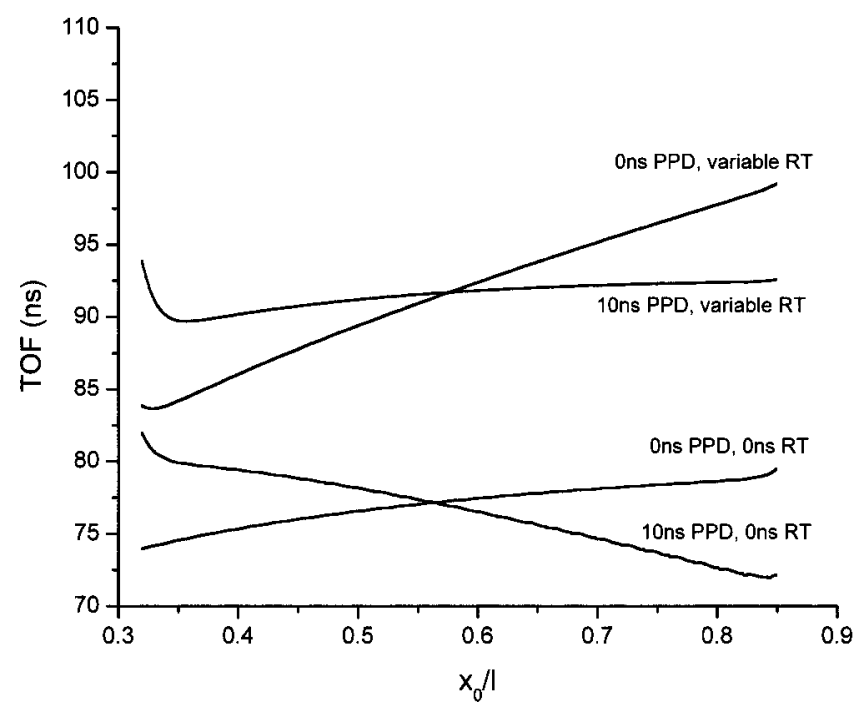

FIG. 8. Simulated times-of-flight at various starting points in the buncher for $V_{b}=1000 \mathrm{~V}$ and $V_{e}=5 \mathrm{eV}$ and at selected values of PPD and RT.

fect of the addition of the pulse propagation with zero rise time is to broaden the distribution of flight times with, as expected, those nearest the exit of the buncher having the longest time-of-flight. The distribution of flight times is similar to that predicted by the simple time-shift formula [Eq. (3) and Fig. 4], though there are important differences. For instance, the particles that start close to the entrance of the buncher arrive earlier than expected. This is caused by the fact that, though the potential may not have physically reached the electrode in which the positron is immersed, the particle begins to feel the electric field as the pulse propagates towards it down the chain. This results in particles temporarily experiencing enhanced acceleration, and this effect is felt strongest where the applied potentials are largest. Detailed comparisons of the speeds of the positrons, and the electric potentials and fields they experience on passing down the buncher, have confirmed that this effect occurs and is, together with the time shift imposed by the delay, responsible for the form of the time-of-flight distribution.

The effect of the variable rise time, for zero pulse propagation delay, is also illustrated in Fig. 7. In this case, those particles nearest the entrance to the buncher have the longest times-of-flight since, as pointed out in Sec. II A, the pulse takes longer to rise to its maximum at higher $x_{0} / l$. The time differences between this curve and that for zero propagation delay and rise time are approximately those due to the rise times at various $x_{0} / l$. It is notable that trends of the curves for pulse propagation delay without rise time and vice versa are very nearly opposite to one another and when the two effects are combined into the simulation leads to a near flat time-of-flight distribution with $x_{0} / l$ as illustrated in Fig. 7. We have also simulated the effect of adding a constant pulse rise time of $20 \mathrm{~ns}$ (with $10 \mathrm{~ns}$ pulse propagation delay) and found it merely to shift the times-of-flight by around half the rise time.

Examples of the effects of initial positron motion in the buncher are given in Fig. 8 for $V_{b}=1 \mathrm{kV}$ and $V_{e}=5 \mathrm{eV}$ (with the motion in the direction of decreasing applied voltage). This corresponds closely to tests performed with the dc

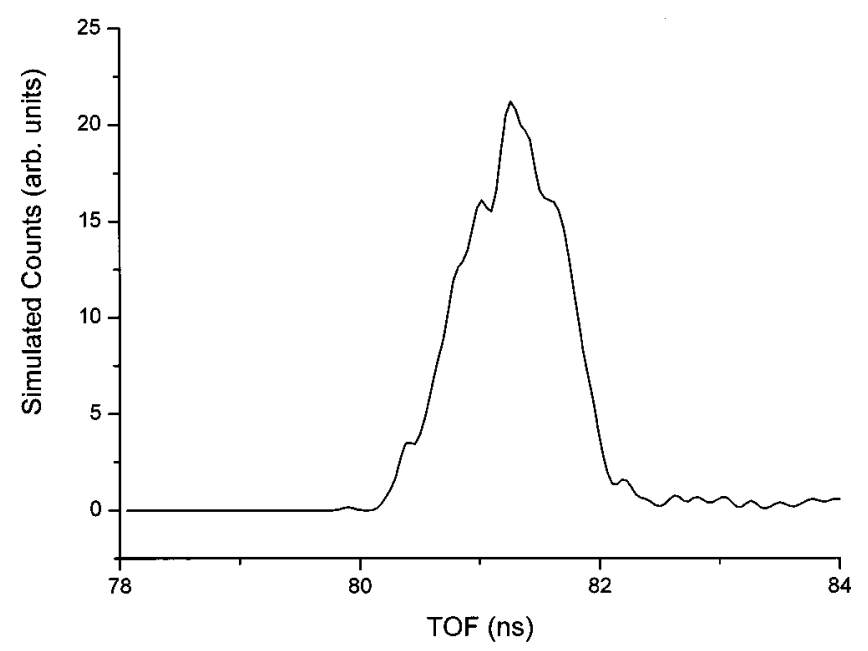

FIG. 9. Simulated time-of-flight distribution of a positron beam with an overall energy spread of $5 \mathrm{eV}$.

positron beam as described in Sec. II B. Overall the features are similar to those shown in Fig. 7 for $V_{e}=0 \mathrm{eV}$. The case for zero pulse propagation delay shows that, as expected, positrons farthest from the exit of the buncher have the longer times-of-flight; however, this situation is reversed when the $10 \mathrm{~ns}$ propagation delay is added. Again, the effect of the variable pulse rise time has a dramatic narrowing effect on the distribution of flight times.

Simulations were performed in an attempt to model the behavior of a beam with finite energy spread, similar to that emitted from the solid argon moderator for $V_{b}=1 \mathrm{kV}$ and with the variable pulse rise time and the $10 \mathrm{~ns}$ pulse propagation delay. For these simulations the axial magnetic field was added and positron starting angles were chosen randomly up to $30^{\circ}$ with respect to the axis. The results are shown in Fig. 9 in the form of a time-of-flight spectrum constructed from the individual times-of-flight of positrons with kinetic energies selected randomly across a Gaussian function centered on $5 \mathrm{eV}$. From here the timing resolution of the main peak was found to be approximately $1.3 \mathrm{~ns}$ FWHM, in excellent accord with the measured value of $1.2 \mathrm{~ns}$.

The sensitivity of the timing response of the buncher to the pulse propagation delay was also investigated. The simulations here were for $V_{b}=1 \mathrm{kV}$ and $V_{e}=5 \mathrm{eV}$ (i.e., a single beam energy), including the variable pulse rise time. The FWHM of the resultant timing distribution is plotted in Fig. 10. The simulated width falls from around $8 \mathrm{~ns}$ at a delay of 4 ns (unphysical in our case) and passes through a sub-ns minimum between delays of 10-14 ns before increasing again. This figure illustrates how critical this parameter is and that the excellent timing performance of the present buncher, as described in Sec. II B was partly fortuitous, since the simulations were a posteriori.

\section{E. Discussion and applications}

We have described a capacitance-coupled buncher capable of producing time focused positron beams. It can operate in the frequency range up to $100 \mathrm{kHz}$, and efficiencies of bunching of a continuous, radioactive source-based, beam in the region of $10 \%$ have been achieved. The timing reso- 


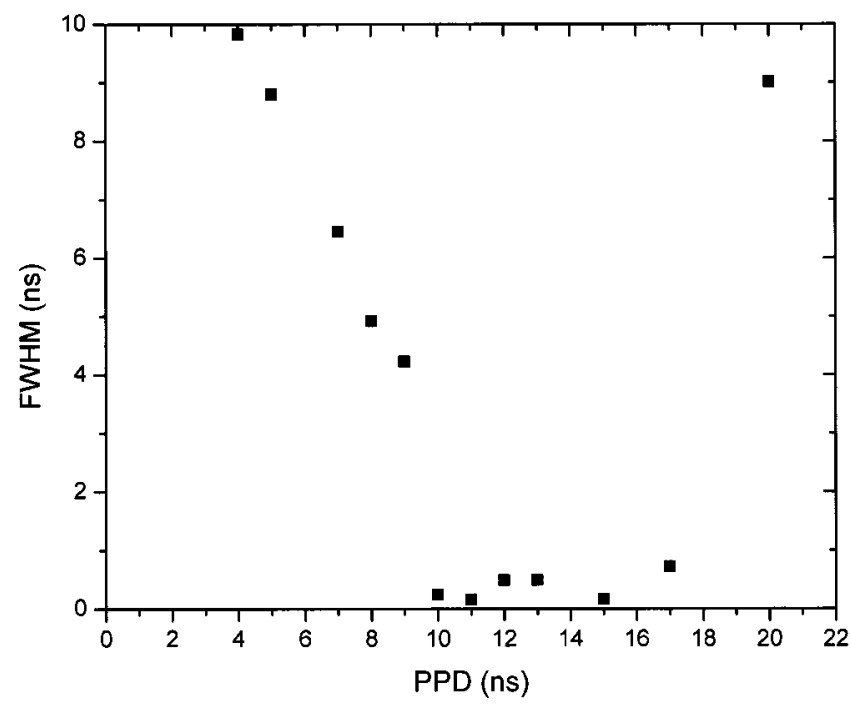

FIG. 10. Values of the timing FWHM as the pulse propagation delay is varied by simulation; see the text for details.

lution of this $1 \mathrm{~m}$ long buncher (see Sec. II B for a detailed description) was found to be less than 2 ns FWHM. The reason for this was traced to the beneficial influence of the propagation time of the voltage pulse down the capacitor chain and the pulse rise time.

There are numerous applications of a buncher of the type described above, both at continuous and pulsed positron sources. Some have already been realized, although many remain for the future. The bunched beam can be used to create a well-defined burst of positronium atoms which can then interact with a laser beam. Positronium spectroscopy ${ }^{17,18}$ is an underdeveloped field and studies of positronium states with principal quantum numbers greater than two appear to be worthwhile, including further work on so-called Rydberg states. ${ }^{31}$ This could lead to advances in laser cooling of positronium. ${ }^{32}$ Mach-Zehnder-type experiments on positronium also seem feasible ${ }^{33,34}$ as do studies of above threshold ionization of this unique species. ${ }^{35}$ A bunched beam can also be used to form positronium atoms in vacuum such that the intrinsic lifetime of the triplet ground state can be measured accurately. The controversy between theory and experiment, and between various experiments, over this parameter has been well documented, ${ }^{36-39}$ and a measurement using a different technique would be worthwhile.

A buncher may be used to inject positrons into an antiproton trap in an effort to promote low energy antihydrogen formation. ${ }^{40-42}$ At Aarhus, the buncher has been used in a separate experiment to inject positron pulses from the microtron beam into a $3 \mathrm{~T}$ magnetic field containing a Penning trap. Stacking of up to 15 slow positron pulses has been observed.

We note that there are some instances when the time focusing device described here cannot be used without the inclusion of a positron remoderator, ${ }^{2}$ bearing in mind the energy spread imparted to the positrons by the bunching process. Firstly, if a monoenergetic timed positron beam is required, for instance for positron/positronium scattering studies, ${ }^{4,5}$ then the extra stage is required. This is also true if the final positron beam is required to be spatially focused in

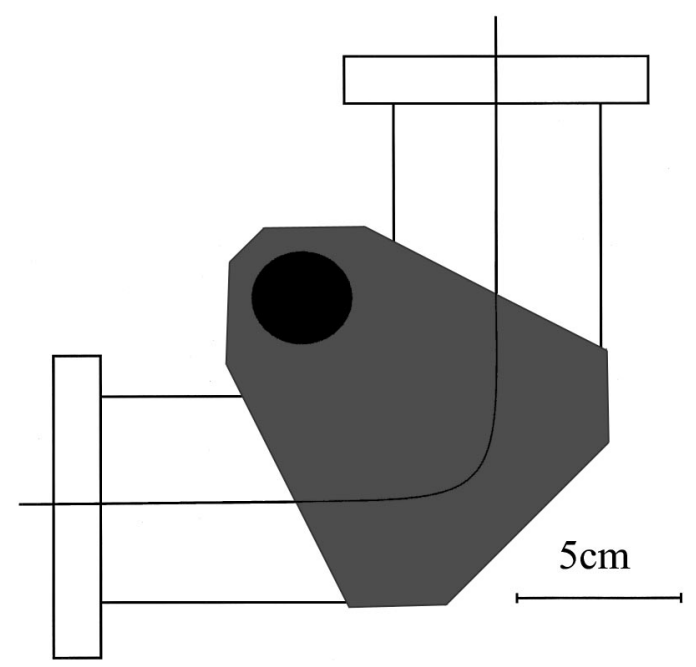

FIG. 11. Side view of one of the plates of the magnetic sector analyzer and its position relative a right-angle bend vacuum chamber. The dark circle represents the yoke on which the 97 turn coil was wound. The plates were $12 \mathrm{~mm}$ thick and $26 \mathrm{~mm}$ apart. The central line represents the central positron trajectory.

the directions perpendicular to its propagation. This can be achieved by electrostatic focusing once the positrons have left the magnetic field used to transport along the buncher, although severe limitations are imposed if use is made of the direct output of the buncher due to the energy spread of the beam.

\section{THE MAGNETIC SECTOR FIELD MOMENTUM ANALYZER}

\section{A. Apparatus details}

Figure 11 shows a schematic illustration of the magnetic sector field analyzer (MSFA). The device was simply constructed from two soft iron pole pieces and a magnetizing coil of 97 turns which was wound on a cylindrical yoke $3 \mathrm{~cm}$ in diameter and $3.5 \mathrm{~cm}$ long. The entire unit was arranged on the outside of a vacuum chamber in which a simple positron extraction and electrostatic lens system was housed. The magnetic field was perpendicular to the positron trajectories. The primary purpose of the analyzer was to deflect the low energy positrons (anticlockwise) through an angle of $90^{\circ}$ to remove them from other, heavier, positively charged particles which were transported through the electrostatic system (see Sec. III C). The magnet was slid over a flattened beam pipe and clamped into position. Magnetic fields up to approximately $5 \mathrm{mT}$ could be generated by passing a current of around 3 A through the coil. Compensation for the Earth's magnetic field was achieved using auxiliary pairs of large Helmholtz coils.

The analyzer was tested using a positron beam generated from a low activity ${ }^{22} \mathrm{Na}$ radioactive source together with a moderator made from overlapping annealed tungsten meshes. The moderator could be biased at voltages up to 1 $\mathrm{kV}$ with respect to the vacuum chamber, thus setting the energy of the beam. The beam was transported to the analyzer (and hence the L-shaped vacuum pipe) using a threeelement einzel lens (see Fig. 1). The beam was detected us- 


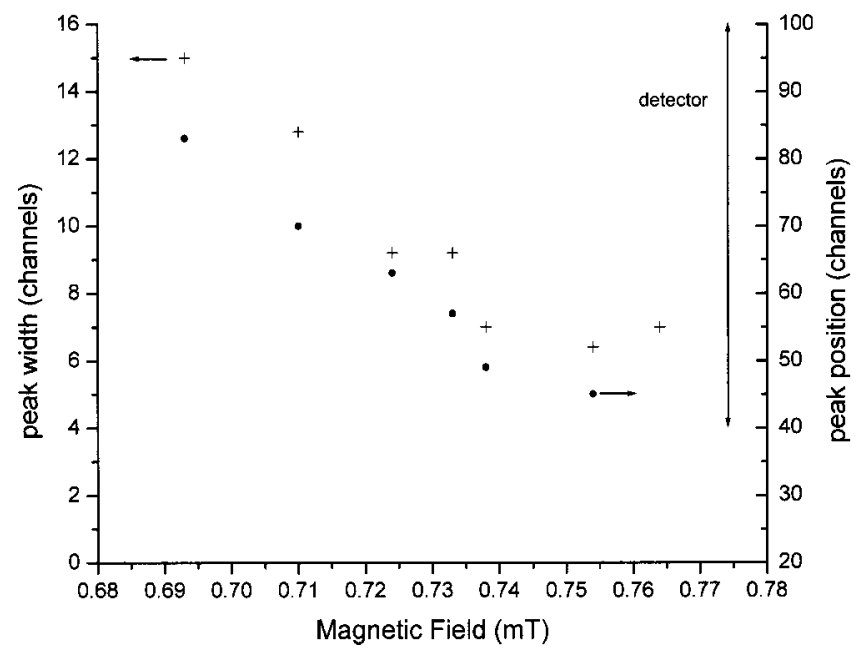

FIG. 12. Position and width of the peak as the low energy positron beam was swept across the position sensitive detector by the application of a magnetic field to the sector analyzer. One channel corresponds to a distance of approximately $0.42 \mathrm{~mm}$.

ing a $25 \mathrm{~mm}$ diam CEMA counter which was position sensitive in one dimension. The detector was arranged such that the axis of position sensitivity coincided with the axis through which the beam would be swept by the applied magnetic field. Two outputs from the CEMA were used to generate the positional data and each was fed through an amplification system, via crossover pickup units, to generate timing information and then into a time-to-amplitude converter. The output of this device was displayed on a multichannel analyzer, from which the position of the peak and its width (which corresponded to the position at which the beam struck the detector and the physical diameter of the beam at the detector, respectively) were read directly.

\section{B. Tests of performance}

Figure 12 shows the position of the peak and its width (in channels, the conversion factor into units of $\mathrm{mm}$ is given in the figure caption) at a fixed beam energy of $1000 \mathrm{eV}$ for various magnetic field settings. Note that the beam was swept across the entire face of the detector as the field was changed by only of the order of $10^{-4} \mathrm{~T}$, at a field of around $10^{-3} \mathrm{~T}$. These data were taken with the central element of the einzel lens set to $-2.5 \mathrm{kV}$ (the two outer elements were grounded). The best focus of the beam obtained was a width of about seven channels, or approximately $3 \mathrm{~mm}$, when the position of the peak was towards the lower channel numbers. This corresponded to deflection of the beam through an angle of greater than $90^{\circ}$ which meant that, for the particular conditions under which these data were taken, the optimum focus was to one side of the detector. The measured width increased steadily as the beam was swept across the detector through the $90^{\circ}$ deflection angle point. However, it was a relatively simple matter to change parameters (einzel lens voltage and magnetic field) slightly to achieve the optimum focus in the center of the detector.

The performance of the system at fixed magnetic field is shown in Fig. 13. As the energy of the beam was varied (by changing the voltage applied to the moderator) between 480

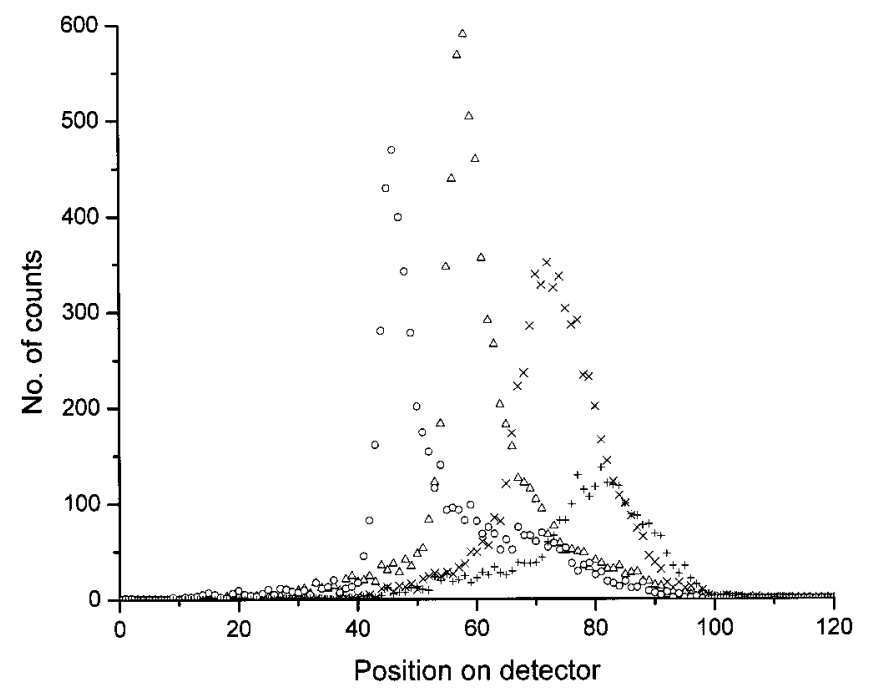

FIG. 13. The positional readout performance of the sector analyzer at four beam energies and a fixed magnetic field of approximately $0.5 \mathrm{mT}[(\bigcirc), 480$ $\mathrm{eV} ;(\triangle), 520 \mathrm{eV} ;(\times), 560 \mathrm{eV} ;(+), 600 \mathrm{eV}]$.

and $600 \mathrm{eV}$ it was swept across the face of the detector and spectra were taken showing the response of the system. At each kinetic energy the einzel lens voltage was changed to maximize the beam intensity on the detector. (However, it was found empirically that optimum focusing and transmission did not always occur at the same einzel lens voltage. More particularly, peak transmission occurred at an einzel lens voltage typically 100-200 V lower than that which produced the best resolution.) As the energy of the beam was raised, the width of the peak increased. This is similar to the observation concerning Fig. 12 and relates to the combination of conditions pertaining to each run. It is also noticeable that the peaks, although having relatively narrow FWHM (particularly those for 480 and $520 \mathrm{eV}$ ) each have broad pedestals, which may be asymmetric. This is probably a result of the relatively large emitting diameter of the positron moderator, which was estimated to be around $5 \mathrm{~mm}$.

\section{Discussion and applications}

In the above subsections we have described the operation of a MSFA as a momentum selector for a low energy positron beam. The device has some important advantages when used as a filter to separate positrons from other species, both charged and neutral, which may be traveling along a common axis with the positrons. First, the instrument is simple to construct and easy to implement. The entire device can be located on the outside of a stainless steel vacuum chamber used to transport the beam; a significant simplification over the usual electrostatic beam deflectors. ${ }^{1,22,25}$ It can be used as part of a compact beam transport system such that, for instance, positrons can be readily removed from line-of-sight with the radioactive source used to produce the beam. The MSFA can be compatible with, as was done in the work reported here, auxiliary electrostatic lens systems. Second, we note that the device is suited for use with positrons of keV-type energies, since the magnetic fields involved are still easily manageable; i.e., of the 1-10 mT. Here it has 
advantages over the use of electrostatic deflectors which typically need static voltages around a factor of 2 greater than the beam energy.

The MFSA is also a true momentum selector, rather than an energy selector, to which class electrostatic instruments belong. Thus, the device can be used to separate positrons from ions which have equal kinetic energies. This attribute was put to use in the experiment of Merrison et al. ${ }^{26,43}$ in which low energy positrons which had been liberated from a positronium atom following capture of the electron by a proton had to be distinguished from the much greater background of low energy protons. More typically it may be necessary to separate energetic positrons from ions which are almost at rest, having been created, for example, as a result of an ionizing collision with a gas atom or molecule. It is straightforward to see that such separation can be accomplished readily at all ionic charge-to-mass ratios at all but a few positron momenta.

The MSFA described here has also been used as a simple beam deflector to operate in a rugged radiation environment near the beam dump of a $100 \mathrm{MeV}$ microtron electron beam used in the ISA positron facility. ${ }^{8}$ The device was used to deflect the remoderated positrons through an angle of approximately $45^{\circ}$, both to facilitate their removal from the incoming positron beam and their injection into a solenoidal magnetic transport field.

\section{ACKNOWLEDGMENTS}

The authors wish to thank the Danish Natural Science Research Council and the EU for collaborative support via the EUROTRAP network (Contract No. ERBFMRX-CT970144). The UWS positron physics program is supported by the EPSRC. They also thank D. L. Adams who wrote the computer program to evaluate the capacitances to insert in the buncher chain.

${ }^{1}$ P. G. Coleman, in Positron Beams and their Applications, edited by P. G. Coleman (World Scientific, Singapore, 2000), pp. 11-40.

${ }^{2}$ P. J. Schultz and K. G. Lynn, Rev. Mod. Phys. 60, 701 (1988).

${ }^{3}$ A. H. Weiss and P. G. Coleman, in Positron Beams and their Applications, edited by P. G. Coleman (World Scientific, Singapore, 2000), pp. 129189.

${ }^{4}$ M. Charlton and J. W. Humberston, Positron Physics (Cambridge University Press, Cambridge, 2001), 454 pp.

${ }^{5}$ G. Laricchia and M. Charlton, in Positron Beams and their Applications, edited by P. G. Coleman (World Scientific, Singapore, 2000), pp. 41-95.

${ }^{6}$ R. Ley, Hyperfine Interact. 109, 167 (1997).

${ }^{7}$ A. P. Mills, Jr., E. D. Shaw, R. J. Chichester, and D. M. Zuckerman, Rev. Sci. Instrum. 60, 825 (1989).

${ }^{8}$ J. P. Merrison, N. Hertel, H. Knudsen, S. Stahl, and E. Uggerhøj, Appl. Surf. Sci. 149, 11 (1999).
${ }^{9}$ P. G. Coleman, T. C. Griffith, and G. R. Heyland, Proc. R. Soc. London, Ser. A 331, 561 (1973).

${ }^{10}$ J. P. Merrison, M. Charlton, and G. Laricchia, Meas. Sci. Technol. 2, 175 (1991).

${ }^{11}$ K. G. Lynn, W. E. Frieze, and P. J. Schultz, Phys. Rev. Lett. 52, 1137 (1984).

${ }^{12}$ G. Laricchia, S. A. Davies, M. Charlton, and T. C. Griffith, J. Phys. E 21, 886 (1988).

${ }^{13}$ W. S. Crane and A. P. Mills, Jr., Rev. Sci. Instrum. 56, 1723 (1985).

${ }^{14}$ R. S. Conti, B. Ghaffari, and T. D. Steiger, Hyperfine Interact. 76, 127 (1993)

${ }^{15}$ T. J. Murphy and C. M. Surko, Phys. Rev. A 46, 5696 (1992).

${ }^{16} \mathrm{H}$. Stoll, in Positron Beams and their Applications, edited by P. G. Coleman (World Scientific, Singapore, 2000), pp. 237-257.

${ }^{17}$ M. S. Fee, A. P. Mills, Jr., S. Chu, K. Danzmann, R. J. Chichester, and D. M. Zuckerman, Phys. Rev. Lett. 70, 1397 (1993).

${ }^{18}$ M. S. Fee, S. Chu, A. P. Mills, Jr., R. J. Chichester, D. M. Zuckerman, E. D. Shaw, and K. Danzmann, Phys. Rev. A 48, 192 (1993).

${ }^{19}$ A. P. Mills, Jr., Nucl. Instrum. Methods Phys. Res. B 192, 107 (2002).

${ }^{20}$ P. M. Platzman and A. P. Mills, Jr., Phys. Rev. B 49, 454 (1994).

${ }^{21}$ N. Jiang and D. M. Schrader, Phys. Rev. Lett. 81, 5113 (1998).

${ }^{22}$ S. M. Hutchins, P. G. Coleman, R. J. Stone, and R. N. West, J. Phys. E 19, 282 (1986).

${ }^{23}$ P. C. Rice-Evans, D. T. Britton, and B. P. Cowan, Appl. Phys. A: Solids Surf. 43, 283 (1987).

${ }^{24}$ A. Goodyear and P. G. Coleman, Meas. Sci. Technol. 6, 415 (1995).

${ }^{25}$ D. J. Day, Ph.D. thesis, University of London, 1993; D. J. Day, M. CharIton, and G. Laricchia, J. Phys. B: At. Mol. Opt. Phys. 34, 3617 (2002).

${ }^{26}$ J. P. Merrison, H. Bluhme, M. Charlton, H. Knudsen, and M. R. Poulsen, Hyperfine Interact. 109, 313 (1997).

${ }^{27}$ L. D. Hulett, Jr., D. L. Donohue, and T. A. Lewis, Rev. Sci. Instrum. 62, 2131 (1991).

${ }^{28}$ D. A. Dahl, SIMION v7.0, Idaho National Engineering and Environmental Lab., Idaho Falls, ID, 2000.

${ }^{29}$ S. J. Gilbert, C. Kurz, R. G. Greaves, and C. M. Surko, Appl. Phys. Lett. 70, 1944 (1997).

${ }^{30}$ R. G. Greaves and C. M. Surko, in New Directions in Antimatter Physics and Chemistry, edited by C. M. Surko and F. A. Gianturco (Kluwer Academic, Dordrecht, 2001), pp. 21-33.

${ }^{31}$ K. P. Ziock, R. H. Howell, F. Magnotta, R. A. Failor, and K. M. Jones, Phys. Rev. Lett. 64, 2366 (1990).

${ }^{32}$ E. P. Liang and C. D. Dermer, Opt. Commun. 65, 419 (1988).

${ }^{33}$ D. W. Keith, C. R. Ekstrom, Q. A. Turchette, and D. E. Pritchard, Phys. Rev. Lett. 66, 2693 (1991).

${ }^{34}$ M. Oberthaler, Nucl. Instrum. Methods Phys. Res. B 192, 129 (2002); see also T. J. Phillips, Hyperfine Interact. 109, 357 (1997).

${ }^{35}$ L. B. Madsen and P. Lambropolous, Phys. Rev. A 59, 4574 (1999).

${ }^{36}$ J. S. Nico, D. W. Gidley, A. Rich, and P. W. Zitzewitz, Phys. Rev. Lett. 65, 1344 (1990).

${ }^{37}$ S. Asai, S. Orito, and N. Shinohara, Phys. Lett. B 357, 475 (1995).

${ }^{38}$ I. B. Khriplovich, I. N. Meshkov, and A. I. Milstein, JINR Rapid Communications 83, 68 (1997).

${ }^{39}$ R. Foot and S. N. Gninenko, Phys. Lett. B 480, 171 (2000).

${ }^{40}$ M. Charlton, J. Eades, D. Horváth, R. J. Hughes, and C. Zimmermann, Phys. Rep. 241, 65 (1994).

${ }^{41}$ M. H. Holzscheiter and M. Charlton, Rep. Prog. Phys. 62, 1 (1999).

${ }^{42}$ M. Amoretti et al., Nature (London) 419, 456 (2002).

${ }^{43}$ J. P. Merrison, H. Bluhme, J. Chevallier, B. I. Deutch, P. Hvelplund, L. V. Jørgensen, H. Knudsen, M. R. Poulsen, and M. Charlton, Phys. Rev. Lett. 78, 2728 (1997). 